\title{
La formation de géodiversité et l'habitation durable de la Terre
}

\author{
Christophe Grenier* \\ Géographie, Université de Nantes, UMR6554 LETG, Nantes, France
}

Reçu le 19 mars 2018. Accepté le 12 septembre 2019

Avant de s'affirmer comme une discipline attachée à «penser l'espace des sociétés humaines », la géographie, notamment sa tradition française, a fait de l'étude des rapports hommes/environnement son objet de prédilection. Ainsi l'école française de géographie a-t-elle forgé, dans différents textes publiés au tournant du $\mathrm{XX}^{\mathrm{e}}$ siècle, la notion de genre de vie pour qualifier la diversité sociale résultant de la lente adaptation des sociétés à leurs environnements. Puis, la géographie a diversifié ses objets d'études et a tendu à oublier cet héritage pour, en matière d'étude des rapports hommes/environnement, céder le plus souvent à la fascination de la puissance de la technique. Cet article témoigne du fait qu'en ces temps de changements globaux, la géographie sait se réinventer et proposer de nouvelles conceptualisations de son objet de prédilection.

La Rédaction

\begin{abstract}
Résumé - L'adaptation à l'environnement et l'isolement sont cruciaux dans la genèse de la géodiversité, ou diversité géographique, comme dans la formation des diversités culturelle et biologique. Ces facteurs, d'ordre évolutionniste et écologique dans le dernier cas, historique et géographique dans les autres, se dégradent avec la mondialisation. Après avoir exposé la conception de la géographie sur laquelle est fondée la notion, on examine la formation de la géodiversité qui accompagne le peuplement humain de la Terre, avant de présenter deux cas où les géographies mises en œuvre par les populations expliquent la diversification linguistique par isolement territorial et l'adaptation optimale d'un genre de vie aux écosystèmes marins. Préserver et inventer la géodiversité permettent l'habitation durable de la Terre pour l'humanité et les autres êtres vivants dans leurs diversités respectives.
\end{abstract}

Mots-clés : environnement / territoire / géographie / adaptation / isolement

Abstract - The forming of geodiversity and the sustainable habitation of Earth. Geodiversity, as evoked in this paper, refers to geographical, not to geological diversity. The interdisciplinary approach adopted here aims to point out that the forming of geodiversity follows the same patterns as biological and cultural diversification processes. Darwinian evolutionism explains biological diversification through Earth's ecology, by the isolation of populations and their adaptation to the environment. But during the peopling of the Earth by human populations, their ways of life - geographical techniques mainly related to habitat, food and mobility - took the place of evolution for Homo sapiens, consequently allowing the active adaptation of human populations through the alteration of their environment and the control of isolation owing to geographically-linked techniques and to territories. Humankind thus enters history as a result of its geographical activity, geography being considered here as the science that studies the footprints (or graphics) produced by human populations on Earth (Geo). This process gives birth to Geodiversity, i.e. the diversity of human geographies at regional scales reflecting the diverse and sustainable ways of living on Earth. The peopling of Austronesian Oceania and its consequent cultural diversification such as the adaptation of the Vezo people to the marine environment of the southwest coast of Madagascar, are examples of how ways of life and territories may produce geodiversity. Preserving and inventing geodiversity provide the means to live sustainably on Earth.

Keywords: environment / territory / geography / adaptation / isolation

\footnotetext{
*Auteur correspondant : christophe.grenier@univ-nantes.fr
} 
L'un des buts que Vidal de la Blache (1899) assignait à la science géographique était d'expliquer la diversité terrestre, ce qui a depuis fait l'objet de nombreux travaux. Est-il alors justifié de poursuivre l'entreprise? Oui, pour plusieurs raisons. D'abord parce que l'intérêt que les géographes portent à la diversité terrestre s'est estompé au cours du $\mathrm{XX}^{\mathrm{e}}$ siècle, sans doute, comme le remarque Claval (1998, p.441), à cause de l'attention exclusive que nombre d'entre eux accordent à l'espace, ce qui «élimine deux des dimensions fondamentales de la réalité géographique: ses aspects physiques et biologiques, et la diversité de l'expérience et du sens que les groupes humains donnent à la Terre ». Or la notion de géodiversité - ou diversité géographiqueprésentée ici est ancrée dans une conception de la géographie qui appréhende la Terre à différentes échelles, dans son unité -le globe, l'écoumène, le Monde $^{1}$ - et dans sa diversité-les écosystèmes, milieux $^{2}$ et espaces ${ }^{3}$ qui en forment la trame régionale.

Ensuite, parce que la racine $G \hat{e}$, commune à plusieurs sciences, fait de "géodiversité» un mot polysémique. Géologues et géomorphologues la définissent comme la «diversité des éléments géologiques, géomorphologiques et pédologiques » (Gray, 2005). Dans ce sens, la notion de géodiversité a été forgée dans les années 1990 par des «géoscientifiques» australiens désireux de conserver le patrimoine géologique (Gray, 2005). Depuis, ce dernier est protégé dans plusieurs pays au sein de « géoparcs »-certains ont le label global geopark de l'UNESCO - accueillant un "géotourisme » qui suscite des tensions entre les deux pôles de la patrimonialisation: héritage à préserver ou ressource à exploiter (Poiraud et Dandurand, 2017). La reconnaissance scientifique et la labellisation internationales de cette géodiversité-là lui confèrent une notoriété qui peut faire paraître vaine la proposition d'utiliser le même mot pour désigner la diversité géographique. Une autre acception du terme a en effet été présentée à la même époque, là aussi en lien avec la conservation de la nature, afin de penser celle-ci à partir de la diversité géographique (Grenier, 2000). Quelques géologues ou géomorphologues citent cette version géographique de la géodiversité (Patzak, 2002; Portal, 2017) que des géographes travaillant sur la conservation de la nature (Mathevet et Poulin, 2006; Héritier et Laslaz, 2008;

\footnotetext{
${ }^{1}$ Le Monde désigne l'extension de l'espace géographique mondial à un moment historique donné du système Monde (Dollfus, 1990).

${ }^{2}$ Milieu est employé dans ce texte dans le sens que lui donne Berque (1986) : «relation d'une société à l'espace et à la nature ».

${ }^{3}$ En géographie, l'espace est à la fois «un produit social organisé et un système de lieux » et, à ce titre, «une portion définie de la surface terrestre» (Brunet, 1992).
}

Laslaz, 2012 ; Mathevet et Marty, 2015) ont utilisée, sans l'avoir précisée. On peut résumer l'intérêt d'étudier cette forme-ci de géodiversité à ce qu'en géographie, Gê ne se réduit pas à la lithosphère mais désigne « la Terre en tant qu'elle est habitée par l'humanité » (Berque, 1996, p. 71).

La pérennité de notre demeure terrestre est la principale raison de continuer à s'intéresser à la diversité géographique un siècle après Vidal de la Blache. Car comme le souligne Robic (1992, p. 149) à propos de ce dernier et de ses contemporains: "Nulle urgence conservationniste ne semble s'imposer à l'opinion de ces scientifiques, pas plus qu'elle ne s'exprime dans une opinion Belle Époque». C'est une différence majeure avec l'époque actuelle, marquée par l'accélération du changement écologique global (Steffen et al., 2007) et les menaces d'effondrement systémique qui en découlent (Servigne et Stevens, 2015), ce dont la communauté scientifique et une part croissante de l'opinion publique sont désormais conscientes. La notion de géodiversité s'inscrit ainsi dans une démarche où la géographie est considérée, à l'instar de la conservation biology, comme une « discipline à mission» (Meine et al., 2006).

Car du $X^{\mathrm{e}}$ siècle à nos jours, la diversité géographique planétaire diminue de plus en plus vite et de façon concomitante à l'érosion de la biodiversité et de la diversité culturelle ${ }^{4}$. Cette dynamique est celle de la mondialisation géographique, ou de la propagation sur la Terre de géographies modernes, c'est-à-dire fonctionnelles pour le capitalisme et/ou pour l'État colonial puis national au cours des périodes historiques de formation du système Monde (Grenier, 2019a). La mondialisation géographique regroupe deux processus. D'une part, l'«ouverture géographique » généralisée, ou la connexion progressive des régions de la Terre au système Monde, entraîne la profonde altération des espaces, milieux et environnements traditionnels de ces régions et s'accompagne de l'aliénation, voire de la destruction des populations et des sociétés qui y vivent. D'autre part, l'homogénéisation géographique découle de la diffusion d'un nombre restreint de types d'espaces et d'environnements reproduits dans diverses régions de la Terre et qui se ressemblent tous au sein de leurs catégories respectives, ainsi que de l'idéologie à vocation universelle qui les justifie.

Il importe alors d'analyser comment la géodiversité s'est formée car son érosion n'est pas irréversible, puisque populations et sociétés peuvent toujours, en

\footnotetext{
${ }^{4}$ Rappelons que la biodiversité désigne la variété du vivant à différentes échelles écologiques d'espace et de temps, et que l'on peut considérer la diversité culturelle comme la variété des idées, us et coutumes qui donnent du sens à la vie des populations au cours de l'histoire de l'humanité, la diversité linguistique étant un indicateur de celle-ci.
} 
principe, mettre en œuvre de nouvelles géographies. Comment? Les notions d'adaptation à l'environnement et d'isolement, qui expliquent depuis Darwin la diversification biologique, sont utilisées par des archéologues et des anthropologues pour rendre compte de la diversification culturelle. Faut-il préciser que l'emprunt de notions de biologie évolutive ou d'écologie pour comprendre des faits sociaux ne signifie pas qu'on naturalise ceux-ci pour autant? Il s'agit d'adopter une approche pluridisciplinaire, d'utiliser un modèle phylogénétique commun à plusieurs sciences dans lequel les adaptations - passives ou évolutives pour la flore et la faune, actives ou historiques pour l'humanité- des populations à la diversité environnementale de la Terre et à leur relatif isolement en son sein contribuent à expliquer leurs diversifications biologique ou culturelle. Mais la démarche suivie est aussi transdisciplinaire car, en partant de l'espace terrestre, l'objectif est de montrer que, pour l'espèce humaine, ces adaptations sont possibles grâce à la diversité des géographies mises en œuvre au cours de l'histoire par les populations et les sociétés ${ }^{5}$. Or les acteurs géographiques ne sont pas mus par des causes naturelles mais sociales.

L'article commence par exposer la définition de la géographie et les quelques concepts de la discipline sur lesquels est fondée la notion de géodiversité. Il examine ensuite la genèse de la diversité géographique qui permet le peuplement de la Terre par Homo sapiens, avant de présenter deux exemples où les géographies mises en œuvre expliquent la diversité culturelle et l'adaptation à l'environnement des populations. Les Austronésiens illustrent le rôle de l'isolement territorial dans la diversification linguistique, à l'échelle du Pacifique Sud comme à celle de l'archipel de Vanuatu. Et le peuple Vezo a produit une géographie qui a longtemps été un modèle d'adaptation aux écosystèmes marins et littoraux du sud-ouest de Madagascar.

\section{Géographie et géodiversité}

Dans l'optique de la géodiversité, la géographie est envisagée comme la science qui analyse, par leur localisation, les graphies - ou les empreintes-que populations et sociétés tracent sur la Terre, Gê. C'est une

\footnotetext{
${ }^{5}$ À l'époque contemporaine, une géographie d'extension planétaire, homogène et destructrice du vivant conditionne aussi les possibilités d'adaptation biologique. Il y a un quart de siècle déjà, le naturaliste Myers (1994, p. 137) écrivait que l'extinction massive de biodiversité en cours signifiait que « de nombreux processus évolutifs qui ont persisté au cours du phanérozoïque pourraient être suspendus, voire terminés», autrement dit que les transformations anthropiques de la Terre pourraient empêcher, entre autres, certaines adaptations biologiques.
}

conception classique. De Vidal de la Blache (1903) à Brunet (1992), les géographes répètent que l'analyse de la localisation ${ }^{6}$ est au fondement de leur science. L'interprétation étymologique de "graphie» comme «empreinte» ou «signe» pour qualifier le résultat des actions humaines sur la face de la Terre - et dont le déchiffrement définit la science « géographique 》-est une idée ancienne: elle a, entre autres, été avancée par des géographes aussi divers que Vidal de la Blache (1903), Dardel (1952) ou Labasse (1966). Mais les géographes s'étant peu préoccupés des conséquences environnementales planétaires de ces "graphies», ils ont délaissé la notion alors que celle d' « empreinte écologique » devenait commune (Wackernagel et Rees, 1999). Enfin, envisager la Terre à la fois comme totalité et comme demeure de l'humanité est une pensée qui traverse l'histoire de la géographie, de Ritter (1852) à Berque (1996). Elle permet de concilier les approches idiographique et nomothétique comme les branches humaine et physique de la discipline, et d'ouvrir de la sorte la géographie à une conscience écologique d'échelle planétaire.

En produisant, organisant et aménageant l'espace à différentes échelles, divers acteurs sociaux transforment les écosystèmes et constituent ainsi des environnements. Un environnement est donc, à la fois et plus ou moins, naturel, puisqu'il a toujours une dimension biophysique, et social, car il est en partie construit par les pratiques et les représentations humaines. Les genres de vie, que Sorre (1948) qualifie d' "assemblages de techniques» et qui «sont des formes actives d'adaptation du groupe humain au milieu » (on dirait aujourd'hui « «...à l'environnement »), ont toujours été parmi les principaux agents géographiques $^{7}$ et, pendant la majeure partie de l'histoire de l'humanité, les seuls. C'est pourquoi un genre de vie est plus précisément un ensemble de «techniques géographiques » qui agissent sur l'environnement des populations et des sociétés. Les géographies opèrent en retour sur le fonctionnement et l'histoire des sociétés qui les produisent: les concepts de territoire - espace approprié par une population ou une société, patrimoine spatial qui leur fournit ressources et identité (Godelier, 1984; Brunet, 1992) -, de milieu et de paysage - « empreintes-matrices » d'une société (Berque, 1986) - expriment ces relations systémiques. Le terme de « géoculture » désigne le versant

\footnotetext{
${ }^{6}$ La localisation est double: la position d'un lieu sur le globe - entité géophysique - est permanente et absolue puisque indiquée par ses coordonnées de latitude et de longitude, tandis que sa situation dans le monde - entité historique - est changeante et relative car définie par rapport à d'autres lieux ou espaces géographiques (Brunet 1992).

${ }^{7}$ Selon Vidal de la Blache (1911, p. 194), « un genre de vie constitué implique une action méthodique et continue, partant très forte, sur la nature, ou, pour parler en géographe, sur la physionomie des contrées ».
} 
idéel d'une géographie, il regroupe les éléments d'une culture qui contribuent à expliquer les empreintes produites (Sauer, 1925), à se les représenter et à les justifier : milieux, paysages et territoires ont une dimension géoculturelle.

Au cours de l'histoire, ces géographies s'inscrivent sur la Terre, c'est-à-dire sur une étendue finie où, quels que soient les critères choisis pour les définir, il n'existe qu'un nombre limité de régions ou de parties de la planète. Ce dernier point est essentiel car il implique la particularité de chaque région terrestre et par conséquent la valeur intrinsèque de chacune, singularité que la notion d' «écorégion» permet d'appréhender. Parmi les principes biogéographiques qui rendent compte des aires de répartition des flores et des faunes comme de leurs fonctionnements écologique et évolutif, l'isolement est primordial (Barbault, 1995; Blondel, 1995): Darwin (1859, [1989, p. 528]) soulignait «[...] l'importance capitale des barrières, soit de terre, soit de mer, qui non seulement séparent mais circonscrivent les diverses provinces zoologiques et botaniques $»$. Dès la fin du $\mathrm{XIX}^{\mathrm{e}}$ siècle, les naturalistes divisent la Terre en provinces biogéographiques de diverses échelles et appellations selon les règnes considérés. Plus d'un siècle après, c'est à la fois pour comprendre et protéger la diversité biologique que des naturalistes proposent la notion d' "écorégion », qui «fusionne le concept écologique d'écosystème et le concept géographique de région » (Loveland et Merchant, 2004, p. 1) et constitue «une aire de n'importe quelle superficie pourvu qu'elle ait une relative homogénéité écosystémique» (Olson et al., 2001). Parmi les 867 écorégions délimitées, on en sélectionne ensuite 238-appelées les «Global 200 »- dont la conservation est jugée prioritaire, et ce dans tous les domaines biogéographiques, terrestres ou marins (Olson et Dinerstein, 2002). Bien que ces décomptes soient arbitraires, ils permettent cependant de comprendre qu' «une écorégion est un endroit qui occupe une position particulière sur la Terre » (Loveland et Merchant, 2004, p. 4) et, par conséquent, qu'il n'en existe qu'un nombre limité. La singularité de chaque écorégion provient de sa position sur le globe, qui détermine une combinaison unique et non reproductible de facteurs écologiques: chaque écorégion abrite une diversité biologique particulière au sein d'un ensemble terrestre fini.

En géographie, une région est à la fois un espace « reconnu comme une structure dans laquelle se déploie un système particulier» (Brunet, 1986, p. 307), et une partie singulière de la Terre - c'est-à-dire du globe et du Monde identifiée comme telle par divers acteurs, en particulier par ceux qui y vivent (Frémont, 1976). Une région géographique coïncide rarement avec une écorégion ${ }^{8}$ mais en fait

\footnotetext{
${ }^{8} \mathrm{Si}$ les limites d'une écorégion ou d'une région géographique sont représentées linéairement sur une carte, il s'agit en réalité de gradients - écotones ou marches.
}

partie ou en comprend plusieurs, et elle occupe en tant que telle une position unique sur le globe. Mais une région a aussi une situation unique dans le Monde, qui provient d'une trajectoire géohistorique particulière, c'est-à-dire de l'ensemble des situations successives que cette région a occupées au cours de l'histoire, d'abord en tant que « mini-système », puis éventuellement comme partie de «systèmes-monde » (Wallerstein, 2006), et enfin au sein de territoires nationaux et du Monde. Une région a donc des caractères environnementaux, spatiaux et géoculturels qui, ensemble, la distinguent d'autres entités géographiques d'échelle semblable. Les régions géographiques sont néanmoins comparables et se prêtent donc à des typologies, car comme le rappelle Brunet (1990, p. 76): «L'espace géographique a des formes, on y découvre des structures [...]. Il en va de même pour les lieux et les contrées: ces œuvres humaines sont toutes différentes, et cependant elles vont par famille, leur production a ses lois ».

La notion de géodiversité fait le lien entre la variété de l'action géographique et la préservation de la diversité terrestre: elle exprime la pluralité des géographies régionales qui font de la Terre un habitat viable, parce que divers, pour les populations et sociétés humaines comme pour les autres êtres vivants. La géodiversité a longtemps résulté de la variété des genres de vie et des géocultures, c'est-à-dire de la diversité des façons d'habiter la Terre et de se représenter le monde, mais aussi de la multitude de territoires qui permettent à ces diversités d'advenir et de se développer. La géodiversité se traduit par des espaces, des milieux et des environnements singuliers, et ces géographies s'évaluent à l'échelle d'une région particulière ou de la Terre: la géodiversité terrestre est la somme des géodiversités régionales à un moment de l'histoire.

\section{De l'évolution à la géographie}

Si l'environnement terrestre conditionne l'évolution et la diversification du vivant, il explique aussi l'émergence des diverses espèces humaines et leur diffusion sur la Terre (Picq, 2005; Coppens, 2010). La capacité de modifier des environnements très divers émancipe cependant Homo sapiens de la sélection naturelle et le fait ainsi entrer dans l'histoire. Selon Godelier (1984, p. 10), en effet, «l'homme a une histoire parce qu'il transforme la nature»; par conséquent, l'homme a une histoire parce qu'il produit une géographie. Découlant de connaissances et de transformations plus ou moins poussées des écosystèmes, l'adaptation des populations humaines à l'environnement est désormais active, elle n'est plus un processus biologique mais géographique.

Les techniques géographiques liées à l'alimentation, à la mobilité et à l'habitat sont les vecteurs du 
peuplement de la Terre par Homo sapiens, car elles prolongent les effets des facteurs écologiques qui mettent en branle ses populations, en leur permettant d'aller sans cesse plus loin. Ainsi les populations qui, sorties d'Afrique, ont longé la côte méridionale du continent asiatique puis son littoral oriental vers le nord et, au-delà, celui du Pacifique américain, ont un genre de vie qualifié de beachcomber (Oppenheimer, 2003), car elles s'alimentent de ressources collectées sur le rivage marin. Il a aussi fallu que certaines populations inventent la navigation hauturière pour passer les bras de mer entre Sunda et Sahul (Green, 1991a; Orliac, 1997). Enfin, d'autres groupes humains ont dû apprendre à s'accommoder du froid pour habiter la Sibérie, ce qui leur a ensuite permis de traverser la Béringie et de peupler l'Amérique.

Expansion humaine sur la Terre et diversification culturelle vont de pair, car l'essor de la culture, caractéristique du paléolithique supérieur (Tattersall, 1999), découle du peuplement de la planète par notre espèce, de l'occupation concomitante de divers environnements, et des multiples adaptations actives que cela a impliqué. Le développement des facultés d'innovation de l'humanité est d'abord lié à sa diversification géographique, qui exprime la variété de ses adaptations à la diversité écologique terrestre. Cela provoque en retour un essor des capacités cognitives et techniques qui renforcent le pouvoir d'action humaine sur l'environnement ${ }^{9}$. La géodiversité est alors très importante à l'échelle de la Terre et elle augmente au fur et à mesure de l'occupation de la plupart des écorégions terrestres par notre espèce.

Mais l'adaptation des populations humaines à la variété des environnements terrestres n'est pas seule en cause dans ce processus de diversification géographique, l'isolement y joue aussi un rôle majeur. Il s'agit du même patron spatial qu'en écologie, où l'isolement est primordial car il engendre la diversification biologique : «Les barrières, en mettant obstacle aux migrations, jouent un rôle aussi important que le temps quand il s'agit des lentes modifications par la sélection naturelle » (Darwin, 1859 [1989, p. 472]). Lévi-Strauss (1971 [2001, p. 157]) reprend à Darwin le terme de «barrières » pour expliquer la diversification culturelle par isolement :

«Pour développer des différences, pour que des seuils permettant de distinguer une culture de ses voisines deviennent suffisamment tranchés, les conditions sont grosso modo les mêmes que celles qui favorisent la

\footnotetext{
9 Vidal de la Blache (1903, p. 221) l'avait compris: «Si l'activité humaine a pu renouveler en partie la physionomie de la Terre, c'est grâce à la composition déjà très variée du monde vivant, qu'avait engendrée une immense évolution antérieure. C'est de la variété éparse autour d'elle que s'est fortifiée l'intelligence humaine».
}

différenciation biologique entre populations : isolement relatif pendant un temps prolongé, échanges limités, qu'ils soient d'ordre culturel ou génétique. Au degré près, les barrières culturelles sont de même nature que les barrières biologiques $»$.

Le modèle phylogénétique ${ }^{10}$ permet de comparer ces processus de diversification biologique et culturelle, qui ont une double concordance géographique: ils sont la conséquence de l'isolement des populations et ils ont souvent lieu dans les mêmes régions. Les régions où coïncident diversités biologique et linguistique issues de l'isolement ont une "diversité bio-culturelle» (Maffi, 2001) qui provient de multiples «spéciations géographiques $"{ }^{11}$ d'échelles locale ou régionale. Or, parce qu'elle résulte avant tout de l'isolement, des genres de vie et de la géoculture des populations qui vivent dans ces régions, la diversité bio-culturelle dépend en définitive de leurs géographies particulières, c'est-à-dire de la géodiversité. Le recours à des notions de biologie évolutive et d'écologie insulaire comme l'analyse des genres de vie, milieux et territorialités de populations qui habitent, à différentes époques, des régions de diverses échelles permettent de le démontrer.

\section{Techniques géographiques, isolement et adaptation}

\section{L’Océanie austronésienne}

Il y a plusieurs décennies que certaines notions de biologie évolutive et d'écologie insulaire inspirent des recherches en archéologie et en anthropologie sur le peuplement et la diversification culturelle de l'aire linguistique océanienne (Sahlins, 1957; Kirch et Green, 1987; Green, 1991a; Kirch, 2000). La diversification linguistique des populations austronésiennes obéit à un modèle fondé sur un processus spatial en deux temps, répété tout au long de leurs itinéraires migratoires dans le Pacifique Sud pendant quelques milliers d'années. Ce modèle, d'ordre phylogénétique, est celui de la radiation adaptative $^{12}$ (Green, 1991b; Kirch, 2010) : l'isolement

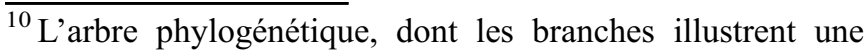
ascendance commune et une diversification, est le modèle fondamental de l'évolutionnisme, utilisé en biologie, en génétique des populations, en linguistique ou en anthropologie, interdisciplinarité qui « lui confère une crédibilité très grande» (Testart, 2012, p. 93). Sur cette approche en sciences humaines, cf. Mace et al., 2005.

${ }^{11}$ Cette notion de biologie évolutive désigne la formation d'une nouvelle espèce par isolement écologique d'une population (Mayr, 1993).

${ }^{12} \mathrm{La}$ radiation adaptative désigne «une rapide évolution de plusieurs espèces issues d'un ancêtre commun qui occupent différentes niches écologiques» (Grant et Grant, 2011, p. 2).
} 
insulaire permet d'abord l'apparition de nouvelles langues, qui se diffusent ensuite avec des migrants vers d'autres îles où elles spécient à nouveau, et ainsi de suite. Ce sont des techniques géographiques - la navigation hauturière et un complexe horticole robuste, autorisant une « domestication progressive de l'inconnu » (Thomas, 2008) - associées à une représentation océanienne du monde agissant comme «support idéologique à l'expansion» (Bachimon, 1995), communes à ces populations austronésiennes, qui leur permettent d'abord de peupler les îles petites et/ou isolées du Pacifique Sud, de s'adapter ensuite à divers environnements en les modifiant parfois de fond en comble, et enfin de se diversifier ainsi par spéciation géographique (Green, 1999; Forestier et Guillaud, 2005; Spriggs, 2010).

La territorialité autorise les populations humaines à agir sur l'isolement, à l'accentuer ou à l'atténuer. L'isolement ou sa rupture découlent en effet de la volonté de repli ou d'expansion territoriale des différentes populations et sociétés, mais aussi de leur désir d'imitation ou de distinction (Lévi-Strauss, 1952). L'extrême diversité linguistique de la Mélanésie s'explique ainsi par des territoires de quelques dizaines de $\mathrm{km}^{2}$ dont les populations entretiennent entre elles des rapports d'hostilité ou des relations d'alliance. Bonnemaison (1986) a montré que l'espace géographique traditionnel de l'archipel de Vanuatu était organisé par des réseaux d'alliances politiques et d'échanges de biens symboliques qui ont créé, grâce à des relations régulières, des «chaînes d'intelligibilité linguistique» entre des communautés possédant chacune sa propre langue. À Vanuatu où l'environnement et la culture sont à peu près semblables pour toutes les populations, celles-ci se distinguent par leur territorialité : la différenciation linguistique de communautés voisines provient de leurs territoires, qui ont ici la fonction des niches écologiques dans la spéciation sympatrique ${ }^{13}$.

C'est grâce à leurs techniques géographiques et géoculture communes que les Austronésiens ont peuplé les îles du Pacifique Sud. Et c'est en raison de leurs adaptations actives à une grande variété d'environnements, de leur isolement et de leurs territoires, autrement dit en raison de leur géodiversité, que les populations austronésiennes se sont ensuite culturellement diversifiées.

\section{Les Vezo}

À l'autre extrémité du monde austronésien, le peuple vezo, qui vit le long du littoral sud-ouest de Madagascar,

\footnotetext{
${ }^{13}$ Dans celle-ci, deux populations d'une même espèce réparties sur une même aire divergent en raison de mécanismes d'isolement qui leur permettent d'occuper des niches écologiques différentes (Mayr, 1993).
}

a longtemps été un exemple d'adaptation durable à l'environnement grâce à un genre de vie aux empreintes légères. L'intérêt du cas tient en outre à ce que la géographie vezo s'inscrit dans une écorégion marine à la forte diversité biologique. Inclus dans l'écorégion «Ouest et Nord de Madagascar» (Spalding et al., 2007), l'un des plus grands systèmes coralliens de la Terre, où se succèdent récifs frangeants, récifs barrières et cayes, borde le littoral sud-ouest de l'île entre les $21^{\circ}$ et $25^{\circ}$ Sud. La grande hétérogénéité écologique et la forte productivité de cette mer côtière, dues aux récifs coralliens, aux mangroves et aux upwellings saisonniers, expliquent qu'elle abritait jadis la diversité biologique la plus riche du domaine marin «Ouest Indo-Pacifique» (Cooke et al., 2003).

Des populations vivent sur ce littoral depuis au moins cinq siècles (Marikandia, 2001); se dénommant ellesmêmes vezo - «ceux qui pagaient»-, elles ont un genre de vie « géodéterministe» (Astuti, 1995), car il implique de vivre sur la plage et de ne tirer ses ressources que de la mer (Fauroux et al., 1992). Ce genre de vie est ancien - en 1595 le navigateur hollandais Houtman signale que les Vezo «ne sèment ni ne moissonnent, ils vivent seulement du poisson» (Engelvin, 1937, p. 65) - et il perdure, bien qu'il soit aujourd'hui menacé par la destruction de l'écosystème corallien régional en raison d'une pêche d'exportation intensive (Grenier, 2013). Si le genre de vie de la société vezo a permis son adaptation durable aux écosystèmes littoraux et marins de la région, c'est que les empreintes produites par ses techniques géographiques d'habitat, d'alimentation et de mobilité ont longtemps été superficielles.

En effet, chez les Vezo, «l'homme s'incorpore de façon frappante à son milieu : la dune et la mer» (Petit, 1923); leur habitat est constitué par «deux rubans, le littoral et la mer côtière » (Engelvin, 1937). Aujourd'hui encore, les villages vezo, bâtis au-dessus de la laisse de mer, comprennent deux ou trois rangées, parallèles à la plage, de cases en bois et roseaux entourées de palissades délimitant les «carrés» familiaux. Pour désigner la pêche, les Vezo emploient un terme équivalent à «maraudage », ce qui souligne l'opportunisme de leurs pratiques et le large spectre des ressources qu'ils exploitent, autrement dit leur adaptation optimale à l'environnement marin. Qu'elles soient destinées à l'autoconsommation, au troc ou à la vente, les espèces ciblées par les Vezo sont très variées (Koechlin, 1975; Iida, 2005; Grenier, 2013). La pirogue à balancier est le trait d'union entre les deux techniques géographiques constitutives du genre de vie vezo, la pêche sur le récif corallien et l'habitat sur la plage. «Moyen d'existence» (Astuti, 1995) des Vezo, la pirogue est la technique géographique centrale de leur genre de vie, qui leur permet de pêcher sur le récif et de se déplacer sur de grandes distances (Cripps et Gardner, 2016), autrement 
dit d'inscrire leur géographie le long du littoral sud-ouest de Madagascar et d'en faire ainsi un espace bien identifié, la région vezo.

C'est donc la géographie des Vezo qui fonde leur identité collective, par leur adaptation à l'environnement littoral et marin, la façon dont ils l'habitent et l'utilisent, les empreintes qu'ils y laissent, les relations qu'ils tissent avec la côte et la mer. L'adaptation à l'environnement est une construction sociale dont la géographie est conjointement l'outil et le facteur de différenciation sur la Terre.

\section{Conclusion : conserver la géodiversité}

A priori, les régions marginales du monde actuel ont, à la fois, la géodiversité la plus élevée - car leur isolement géographique a permis d'en préserver l'essentiel des singularités écologique et/ou géoculturelleet le potentiel d'invention de géodiversité le plus important "parce qu'elles constituent un espace d'autonomie indispensable à la création d'alternatives systémiques»" (Servigne et Stevens, 2015, p. 195). À l'heure où «tout peut s'effondrer»-réchauffement climatique accéléré, érosion rapide des diversités écologique et biologique, destructions massives d'habitats terrestres et marins, pollutions intenses des sols, des mers, des eaux continentales et de l'atmosphère, pandémies...-, il est vital de conserver notre habitat terrestre dans sa diversité.

C'est pourquoi rechercher la seule coviabilité socioécologique ne suffit pas, car cela n'impose pas de cultiver - au sens de prendre soin et d'augmenter - la diversité géographique (Grenier, 2019b). La genèse de la géodiversité montre au contraire qu'elle est une production de géographies permettant aux diversités biologique et culturelle régionales d' «avoir lieu» ensemble, ce qui implique des territoires où elles puissent advenir et durer. Conservation et création de géodiversité reposent donc en définitive sur la disponibilité d'espaces appropriés comme sur le pouvoir de maîtriser leur connexion au système Monde, de façon à garder la possibilité de spéciations géographiques, de préservation ou d'invention de géographies aux empreintes légères. L'enjeu est de préserver et même d'accroître l'habitabilité de la planète, c'est-à-dire la qualité de l'écoumène et de la biosphère pour l'humanité et le vivant dans leurs diversités respectives.

\section{Références}

Astuti R., 1995. People of the sea. Identity and descent among the Vezo of Madagascar, Cambridge, Cambridge University Press.

Bachimon P., 1995. L'insularité océanienne dans la cosmogonie maohi, L'Espace géographique, 24, 3, 227-235.
Barbault R., 1995. Écologie des peuplements. Structure et dynamique de la biodiversité, Paris, Masson.

Berque A., 1986. Le sauvage et l'artifice. Les Japonais devant la nature, Paris, Gallimard.

Berque A., 1996. Etre humains sur la Terre. Principes d'éthique de l'écoumène, Paris, Gallimard.

Blondel J., 1995. Biogéographie. Approche écologique et évolutive, Paris, Masson.

Bonnemaison J., 1986. Les fondements d'une identité. Territoire, histoire et société dans l'archipel de Vanuatu (Mélanésie), Livre I, L'arbre et la pirogue, Bondy, Orstom.

Brunet R., 1986. L'espace, règles du jeu, in Auriac F., Brunet R. (Eds), Espaces, jeux et enjeux, Paris, Fondation Diderot/ Fayard, 297-316.

Brunet R., 1990. Le déchiffrement du monde, in Brunet R., Dollfus O. (Eds), Mondes nouveaux, Paris, Hachette, 9-271.

Brunet R., 1992. Entrées «espace», «géographie», «position», «situation», «territoire», in Brunet R., Ferras R., Théry H., Les mots de la géographie. Dictionnaire critique, Montpellier/Paris, RECLUS/La Documentation française.

Claval P., 1998. Histoire de la géographie française de 1870 à nos jours, Paris, Nathan.

Cooke A., Lutjeharms J.R.E., Vasseur P., 2003. Marine and coastal ecosystems, in Goodman S.M., Benstead J.P. (Eds), The natural history of Madagascar, Chicago, The University of Chicago Press, 179-209.

Coppens Y., 2010. L'histoire de l'homme, Paris, Odile Jacob.

Cripps G., Gardner C., 2016. Human migration and marine protected areas: insights from Vezo fishers in Madagascar, Geoforum, 74, 49-62, http://dx.doi.org/10.1016/j.geofo rum.2016.05.010.

Dardel E., 1952. L'homme et la terre, Paris, Presses universitaires de France.

Darwin C., 1859. On the origin of species by means of natural selection or the preservation of favoured races in the struggle for life, London, John Murray. Trad. fr. : L'origine des espèces au moyen de la sélection naturelle ou la lutte pour l'existence dans la nature, Paris, La Découverte, 1989.

Dollfus O., 1990. Le Système Monde, in Brunet R., Dollfus O. (Eds), Mondes nouveaux, Paris, Hachette.

Engelvin A., 1937. Les Vézos ou "Enfants de la mer», Bellevue, Librairie Vincentienne et Missionnaire.

Fauroux E., Laroche J., Marikandia M., 1992. Brève esquisse d'une description de la société vezo (littoral occidental de Madagascar) à la fin du $\mathrm{XX}^{\mathrm{e}}$ siècle. Document de travail, Tuléar, ERA, CNRE ORSTOM/IFSH.

Forestier H., Guillaud D., 2005. Des Austronésiens en AsiePacifique. Continuités et ruptures sur le chemin des migrations anciennes, Aséanie, 16, 11-40.

Frémont A., 1976. La région, espace vécu, Paris, Presses universitaires de France.

Godelier M., 1984. L'idéel et le matériel. Pensée, économies, sociétés, Paris, Fayard.

Grant P.R., Grant B.R., 2011 [1 $1^{\text {st }}$ ed. 2007]. How and why species multiply? The radiation of Darwin's finches, Princeton, Princeton University Press. 
Gray M., 2005. Geodiversity and geoconservation: what, why, and how?, Parks Stewardship Forum, 22, 3, 4-12.

Green R.C., 1991a. Near and remote Oceania: disestablishing "Melanesia" in culture history, in Pawley A. (Ed.), Man and a half: essays in Pacific anthropology and ethnobiology in honour of Ralph Bulmer, Auckland, Polynesian Society, 491-502.

Green R.C., 1991b. The Lapita cultural complex: current evidence and proposed model, in Bellwood P. (Ed.), IndoPacific prehistory 1990: proceedings of the $14^{\text {th }}$ Congress of the Indo-Pacific Prehistory Association (IPPA), 26 August2 September, Yogyakarta (Indonesia), Canberra, IPPA, 295305.

Green R.C., 1999. Integrating historical linguistics with archeology: insights from research in remote Oceania, Bulletin of the Indo-Pacific Prehistory Association, 18, 3-16.

Grenier C., 2000. Conservation contre nature. Les îles Galápagos, Paris, IRD.

Grenier C., 2013. Genre de vie vezo, pêche «traditionnelle» et mondialisation sur le littoral sud-ouest de Madagascar, Annales de Géographie, 693, 549-571, www.cairn.info/ revue-annales-de-geographie-2013-5-page-549.htm.

Grenier C., 2019a. De la géographie de la mondialisation à la mondialisation géographique, Annales de Géographie, 726, $58-80$.

Grenier C., 2019b. A geographical approach to socioecological coviability, in Barrière O., Behnassi M., David G., Douzal V., Fargette M., Libourel T., Loireau M., Pascal L., Prost C., Ravena Canete V., Seyler F., Morand S. (Eds), Coviability of social ecological systems: reconnecting mankind to the biosphere in an era of global change. Vol. 1 The foundations of a new paradigm, Cham, Springer, 253268.

Héritier S., Laslaz L. (Eds), 2008. Les parcs nationaux dans le monde, Paris, Ellipses.

Iida T., 2005. The past and present of coral reef fishing economy in Madagascar: implications for the selfdetermination of resource use, in Kishigami N., Savelle J.M. (Eds.), Indigenous use and management of marine resources, Senri Ethnological Studies, 67, 237-258.

Kirch P.V., 2000. On the road of the winds. An archeological history of the Pacific islands before European contact, Berkeley, University of California Press.

Kirch P.V., 2010. Peopling of the Pacific: a holistic anthropological perspective, Annual Review of Anthropology, 39, 131-148, http://dx.doi.org/10.1146/annurev. anthro.012809.104936.

Kirch P.V., Green R.C., 1987. History, phylogeny, and evolution in Polynesia, Current Anthropology, 28, 4, 431-456.

Koechlin B., 1975. Les Vezo du sud-ouest de Madagascar. Contribution à l'étude de l'écosystème de semi-nomades marins, Paris, Mouton/EPHE.

Labasse J., 1966. L'organisation de l'espace. Éléments de géographie volontaire, Paris, Hermann.

Laslaz L. (Ed.), 2012. Atlas mondial des espaces protégés. Les sociétés face à la nature, Paris, Autrement.
Lévi-Strauss C., 1952. Race et histoire, in Lévi-Strauss C., 2001. Race et histoire. Race et culture, Paris, Albin Michel/ UNESCO.

Lévi-Strauss C., 1971. Race et culture, in Lévi-Strauss C., 2001. Race et histoire. Race et culture, Paris, Albin Michel/ UNESCO.

Loveland T.R., Merchant J.M., 2004. Ecoregions and ecoregionalization: geographical and ecological perspectives, Environmental Management, 34, suppl. 1, 1-13, http:// dx.doi.org/10.1007/s00267-003-5181-x.

Mace R., Holden C., Shennan S. (Eds), 2005. The evolution of cultural diversity. A phylogenetic approach, London, UCL Press.

Maffi L. (Ed.), 2001. On biocultural diversity. Linking language, knowledge, and the environment, Washington (DC), Smithsonian Institution Press.

Marikandia M., 2001. The Vezo of the Fiherena Coast, Southwest Madagascar: yesterday and today, Ethnohistory, 48, 1-2, 157-170, http://dx.doi.org/10.1215/00141801-48$1-2-157$.

Mathevet R., Poulin B., 2006. De la biologie à la géographie de la conservation, Bulletin de l'Association de Géographes Français, 3, 341-354.

Mathevet R., Marty P., 2015. La géographie de la conservation : entrevoir, voir et porter attention à la biodiversité, in Mathevet R., Godet L. (Eds), Pour une géographie de la conservation. Biodiversités, natures et sociétés, Paris, L'Harmattan, 35-62.

Mayr E., 1993. Darwin et la pensée moderne de l'évolution, Paris, Odile Jacob.

Meine C., Soulé M., Noss R.F., 2006. «A mission-driven discipline $»$ : the growth of conservation biology, Conservation Biology, 20, 3, 631-651, http://dx.doi.org/10.1111/ j.1523-1739.2006.00449.x.

Myers N., 1994. Global diversity: losses, in Meffe G.K., Carroll C.R., (Eds), Principles of conservation biology, Sunderland (MA), Sinauer Associates, 110-140.

Olson D.M., Dinerstein E., Wikramanayake E.D., Burgess N. D., Powell G.V.N., Underwood E.C., D'amico J.A., Itoua I, Strand H.E., Morrison J.C., Loucks C.J., Allnutt T.F., Ricketts T.H., Kura Y., Lamoreux J.F., Wettengel W.W., Hedao P., Kassem K.R., 2001. Terrestrial ecoregions of the world: a new map of life on Earth, Bioscience, 51, 11, 933938, http://dx.doi.org/10.1641/0006-3568(2001)051[0933: TEOTWA]2.0.CO;2.

Olson D.M., Dinerstein E., 2002. The Global 200: priority ecoregions for global conservation, Annals of the Missouri Botanical Garden, 89, 2, 199-224, http://dx.doi.org/ 10.2307/3298564.

Oppenheimer S., 2003. Out of Africa's Eden. The peopling of the world, Cape Town, Jonathan Ball Publishers.

Orliac M., 1997. L'homme marin, in Vigne J.-D. (Ed.), Îles. Vivre entre ciel et mer, Paris, MNHN/Nathan, 39-53.

Patzak M., 2002. Tourism and geodiversity: the case of geoparks, in Di Castri F., Balaji V., (Eds), Tourism, biodiversity and information, Leiden, Backhuys Publishers, 319-327.

Petit G., 1923. La vie sur les côtes de Madagascar et l'industrie indigène de la pêche, Annales de Géographie, 176, 142- 
164, www.persee.fr/doc/geo_0003-4010_1923 num_32_176_9975.

Picq P., 2005. Les origines de l'homme. L'odyssée de l'espèce, Paris, Seuil/Tallandier.

Poiraud A., Dandurand G., 2017. De la géoconservation au géotourisme: un glissement de paradigme, Annales de Géographie, 717, 625-653, www.cairn.info/revue-annalesde-geographie-2017-5-page-625.htm.

Portal C., 2017. Paysages géopittoresques et modélisation spatiale des géoparcs européens : vers de nouveaux espaces naturels et culturels protégés, Annales de Géographie, 717, 654-674, www.cairn.info/revue-annales-de-geographie2017-5-page-654.htm.

Ritter C., 1852. Einleitung zur allgemeinen vergleichenden geographie: und Abhandlungen zur Begründung einer mehr wissenschaftlichen Behandlung der Erdkunde. Trad. fr. : Introduction à la géographie générale comparée. Essais sur les fondements d'une géographie scientifique, Paris, Les Belles Lettres, 1974.

Robic M.-C., 1992. Géographie et écologie végétale: le tournant de la Belle Époque, in Robic M.-C. (Ed.), Du milieu à l'environnement. Pratiques et représentations du rapport homme/nature depuis la Renaissance, Paris, Economica, 125-165.

Sahlins M.D., 1957. Differentiation by adaptation in Polynesian societies, The Journal of the Polynesian Society, 66, 3, 291-300.

Sauer C., 1925. The morphology of landscape, in Leighly J. (Ed.), 1969. Land and life. A selection from the writings of Carl Ortwin Sauer, Berkeley, University of California Press, 315-350.

Servigne P., Stevens R., 2015. Comment tout peut s'effondrer. Petit manuel de collapsologie à l'usage des générations présentes, Paris, Seuil.

Sorre M., 1948. La notion de genre de vie et sa valeur actuelle, Annales de Géographie, 306, 97-108, www.persee.fr/doc/ geo_0003-4010_1948_num_57_306_12204.

Spalding M.D., Fox H.E., Allen G. Z.A., Finlayson M., Halpern B.S., Jorge M.A., Lombana A.,
Lourie S.A., Martin K.D., McManus E., Molnar J., Recchia C.A., Robertson J., 2007. Marine ecoregions of the world: a bioregionalization of coastal and shelf areas, Bioscience, 57, 7, 573-583, http://dx.doi.org/10.1641/ B570707.

Spriggs M., 2010. Geomorphic and archaeological consequences of human arrival and agricultural expansion on Pacific islands: a reconsideration after 30 years of debate, in Haberle S., Stevenson J., Prebble M. (Eds), Altered ecologies. Fire, climate and human influence on terrestrial landscapes, Canberra, Australian National University Press, 239-252.

Steffen W., Crutzen P.J., McNeill J.R, 2007. The Anthropocene: are humans now overwhelming the great forces of nature?, Ambio, 36, 8, 614-621, http://dx.doi.org/10.1579/ 0044-7447(2007)36[614:TAAHNO]2.0.CO;2.

Tattersall I., 1999. L'émergence de l'homme. Essai sur l'évolution et l'unicité humaine, Paris, Gallimard.

Testart J., 2012. Avant l'histoire. L'évolution des sociétés, de Lascaux à Carnac, Paris, Gallimard.

Thomas T., 2008. The long pause and the last pulse: mapping East Polynesian colonisation, in Clark G., Leach B.F., O'Connor S. (Eds), Islands of inquiry. Colonisation, seafaring and the archeology of maritime landscapes, Canberra, Australian National University Press, 97-112.

Vidal de la Blache P., 1899. Leçon d'ouverture du cours de géographie, Annales de Géographie, 38, 97-109, https://www. persee.fr/doc/geo_0003-4010_1899_num_8_38_6051.

Vidal de la Blache P., 1903. La géographie humaine, ses rapports avec la géographie de la vie, Revue de Synthèse historique, 7, 219-240.

Vidal de la Blache P., 1911. Les genres de vie dans la géographie humaine, Annales de Géographie, 111, 193212, www.persee.fr/doc/geo_0003-4010_1911_ num_20_111_7340.

Wackernagel M., Rees W., 1999. Notre empreinte écologique, Montréal, Écosociété.

Wallerstein I., 2006. Comprendre le monde. Introduction à l'analyse des systèmes-monde, Paris, La Découverte.

Citation de l'article : Grenier C. La formation de géodiversité et l'habitation durable de la Terre. Nat. Sci. Soc. 28, 1, 3-11. 